\title{
Improving High-resolution Impedance Manometry Using Novel Viscous and Super-viscous Substrates in the Supine and Upright Positions: A Pilot Study
}

\author{
Uni Wong, ${ }^{1,2}$ Erik B Person, ${ }^{1,2}$ Donald 0 Castell, ${ }^{3}$ Erik von Rosenvinge, ${ }^{1,2}$ Jean-Pierre Raufman, ${ }^{1,2}$ and Guofeng Xie ${ }^{1,2 *}$ \\ ${ }^{1}$ Division of Gastroenterology and Hepatology, University of Maryland School of Medicine, Baltimore, MD, USA; ${ }^{2}$ Veterans Affairs Maryland \\ Health Care System, Baltimore, MD, USA; and ${ }^{3}$ Division of Gastroenterology and Hepatology, Medical University of South Carolina College of \\ Medicine, Charleston, SC, USA
}

\section{Background/Aims}

Swallows with viscous or solid boluses in different body positions alter esophageal manometry patterns. Limitations of previous studies include lack of standardized viscous substrates and the need for chewing prior to swallowing solid boluses. We hypothesize that high-resolution impedance manometry (HRiM) using standardized viscous and super-viscous swallows in supine and upright positions improves sensitivity for detecting esophageal motility abnormalities when compared with traditional saline swallows. To establish normative values for these novel substrates, we recruited healthy volunteers and performed HRiM.

\section{Methods}

Standardized viscous and super-viscous substrates were prepared using "Thick-It" food thickener and a rotational viscometer. All swallows were administered in 5-mL increments in both supine and upright positions. HRiM metrics and impedance (bolus transit) were calculated. We used a paired two-tailed $t$ test to compare all metrics by position and substrate.

\section{Results}

The 5-g, 7-g, and 10-g substrates measured 5000, 36200 , and $64700 \mathrm{mPa} \cdot \mathrm{sec}$, respectively. In 18 volunteers, we observed that the integrated relaxation pressure was lower when upright than when supine for all substrates $(P<0.01)$. The 10-g substrate significantly increased integrated relaxation pressure when compared to saline in the supine position $(P<0.01)$. Substrates and positions also affected distal contractile integral, distal latency, and impedance values.

\section{Conclusions}

We examined HRiM values using novel standardized viscous and super-viscous substrates in healthy subjects for both supine and upright positions. We found that viscosity and position affected HRiM Chicago metrics and have potential to increase the sensitivity of esophageal manometry.

(J Neurogastroenterol Motil 2018;24:570-576)

\section{Key Words}

Esophageal motility disorders; Manometry; Viscous substrates

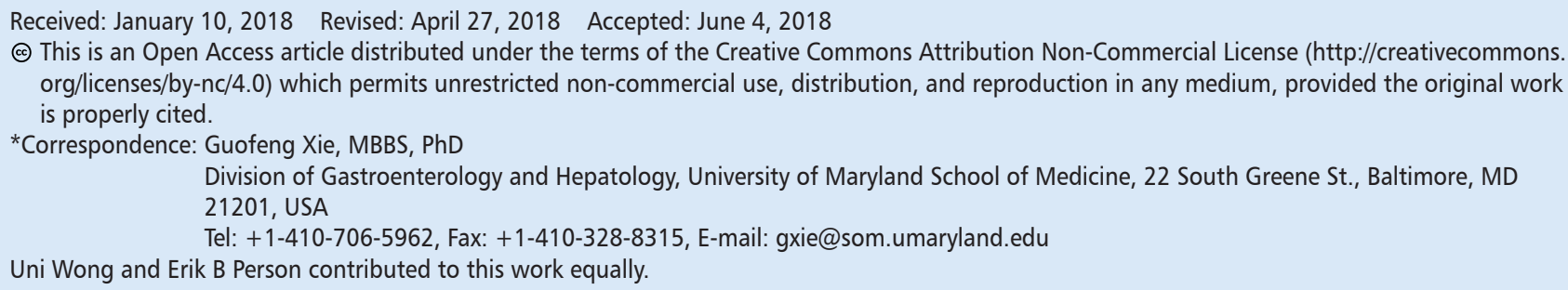




\section{Introduction}

High-resolution esophageal manometry (HRM) is the current gold standard test for diagnosing esophageal motility disorders. ${ }^{1,2}$ The Chicago classification manometric diagnosis is based exclusively on HRM data derived from 10 liquid (normal saline) swallows performed in the supine position. ${ }^{3-7}$ High-resolution impedance manometry (HRiM) catheters (Diversateck Healthcare Inc, Milwaukee, WI, USA) incorporate impedance and manometry sensors, providing information on esophageal peristaltic patterns and pressures as well as whether a bolus transit is complete. The protocol for HRiM testing consists of the patient swallowing ten 5-mL saline and ten 5-mL viscous solutions (Diversateck) in supine position. ${ }^{8}$ Although viscous manometry and impedance values have not been integrated into the current Chicago classification, viscous impedance has been shown to be an important indicator of abnormal motility. ${ }^{9}$ In a retrospective analysis, Clayton et $\mathrm{al}^{9}$ showed that viscous impedance is the most sensitive indicator for detecting subtle esophageal function abnormalities independent of esophageal contractility.

Evaluating swallows with viscous or solid boluses in different body positions has been shown to alter esophageal manometry patterns and may increase sensitivity of motility studies. ${ }^{9-13}$ Some patients with normal manometry studies with liquid swallows have been demonstrated to have diffuse esophageal spasm with solid food ingestion. ${ }^{14}$ Furthermore, upright bread swallows can reveal motility abnormalities overlooked by supine saline swallows, particularly in patients with complaints of non-cardiac chest pain and gastroesophageal reflux disease. ${ }^{10}$ While previous studies have used solid swallows, they lack standardization and their findings may be difficult to reproduce given the foods used. In addition, while upright swallowing may elicit subtle gastroesophageal junction outflow obstruction patterns on manometry, adjoining bolus transit abnormalities noted on impedance have not been properly evaluated.

We hypothesize that performing HRiM using standardized novel viscous and super-viscous swallows in the supine and upright positions will improve sensitivity for detecting esophageal motility abnormalities when compared with traditional saline swallows. Here, we report HRiM values obtained for these substrates in healthy volunteers.

\section{Materials and Methods}

The study was approved by the Institutional Review Board at the University of Maryland, Baltimore (IRB No. 00061276). After obtaining informed consent, a Diversateck HRiM catheter was placed into the esophagus and advanced to the lower esophageal sphincter in the standard fashion. Twenty-one healthy volunteers were recruited from the Baltimore VA Medical Center and the University of Maryland School of Medicine. Exclusion criteria included: (1) age less than 18 or older than 75 years, (2) body mass index $>35$, (3) previously diagnosed gastroesophageal reflux disease or other esophageal motility abnormalities, (4) current use of acid suppression medications, (5) current use of any medications that affect gastrointestinal motility or perception, (6) dysphagia with either solids or liquids or both, and (7) pregnancy.

Viscous and super-viscous substrates were prepared by mixing $100 \mathrm{~mL}$ saline in a glass beaker with a stir bar with 5, 7, or $10 \mathrm{~g}$ of original gluten free "Thick-It" food thickener (Precision Foods, Inc, St. Louis, MO, USA) and then heating them up to the boiling point. The substrates were chosen based on viscosity and solubility. First, we determined that 10 grams are the maximal amount of "Thick-It" substrate that could make uniform viscous solution in $100 \mathrm{~mL}$ normal saline under boiling conditions. We then chose 5 grams as a second experimental group for comparison purposes. After we observed that 3 subjects exhibited oropharyngeal transfer difficulty with the 10 -g super viscous solution, we decided to decrease the 10-g "Thick-It" amount. We tested with 9-g, 8-g, and 7-g solutions, and found that the 7-g solution was the easiest to swallow without oropharyngeal transfer difficulty. We measured the viscosity of each substrate with a digital rotational viscometer (Model NDJ-9s; Shanghai Longway Optical Instruments, Co, Ltd, Shanghai, China) in $\mathrm{mPa} \cdot \mathrm{sec}$ at room temperature. We either used these substrates on the same day or stored them in a $100-\mathrm{mL}$ sterile container in a refrigerator for use within 1 week.

Volunteers first received either 10 (for the first 10 subjects) or 5 (for the last 8 subjects) supine saline swallows, followed by five $5-\mathrm{g}$ swallows, then either five 7-g swallows or five 10-g swallows while supine. Subjects then received 5 saline (for only the last 8 subjects), five $5-\mathrm{g}$, and then either five 7 -g or five $10-\mathrm{g}$ swallows while upright. Because 3 subjects experienced oropharyngeal transfer difficulties with the super-viscous 10 -g substrate, we replaced it with the 7 -g substrate for all subjects after subject \#13. A total of 21 volunteers enrolled and 3 were excluded (see Results section). There were a total of 140 supine saline swallows from 18 subjects; 90 supine 5 -g swallows from 18 subjects; 40 supine 7-g swallows from 8 subjects; 45 supine 10 -g swallows from 9 subjects; 40 upright saline swallows from 8 subjects; 90 upright 5 -g swallows from 18 subjects; 40 upright 7-g swallows from 8 subjects and 45 upright 10 -g swallows 
from 9 subjects. Swallows were performed 30 seconds apart as per standard manometry testing protocol. Integrated relaxation pressure (IRP), distal contractile integral (DCI), distal latency (DL), peristaltic breaks (PB), and bolus transit were measured. We calculated medians, means, standard deviations, 5th, and 95th percentiles for each group. Impedance was analyzed using recovery of tracing to $\geq 50 \%$ of baseline at 5,10 , and $15 \mathrm{~cm}$ above the lower esophageal sphincter. Impedance results were noted as $\%$ of complete swallows/ total swallows. Paired two-tailed $t$ test was used to compare all metrics by positions and substrates to supine saline swallows.

\section{Results}

The 5-g, 7-g, and 10-g viscous substrates measured 5000 $\mathrm{mPa} \cdot \mathrm{sec}$ at $21.6^{\circ} \mathrm{C}, 36200 \mathrm{mPa} \cdot \mathrm{sec}$ at $21.4^{\circ} \mathrm{C}$, and $64700 \mathrm{mPa} \cdot \mathrm{sec}$ at $22^{\circ} \mathrm{C}$, respectively. In comparison, Diversateck proprietary viscous solution measured $36700 \mathrm{mPa} \cdot \mathrm{sec}$ at $22^{\circ} \mathrm{C}$. After testing 13 subjects, 3 subjects experienced oropharyngeal transfer difficulties with the super-viscous $10-\mathrm{g}$ substrate, so it was replaced with the 7-g substrate.

Among the 21 volunteers enrolled, 3 were excluded ( 2 had significant double swallowing and 1 could not tolerate the manometry probe). The remaining 18 volunteers consisted of 10 men and 8 women with an average age of $35.3 \pm 10.6$ years (mean $\pm \mathrm{SD}$ ).
Average body mass index was $25.7 \pm 3.9 \mathrm{~kg} / \mathrm{m}^{2}$ (mean $\pm \mathrm{SD}$ ). Eight were Caucasian, 8 were Asian American, and 2 were African American. No adverse event, including perforation, bleeding or aspiration, was reported by any of the study subjects after the procedures. Examples of HRiM studies are shown in Figure 1.

As shown in Table 1, IRP in the supine position was not significantly different when testing with 5 -g or 7 -g viscous solutions. However, IRP was higher when testing with super-viscous $10-\mathrm{g}$ solution in comparison with saline $(P<0.01)$. When compared to testing with saline in the supine position, IRP was significantly lower in the upright position with all substrates including saline $(P$ $<0.01)$, 5-g $(P<0.01)$, 7-g $(P<0.01)$, and 10-g $(P<0.01)$.

As shown in Table 2, DCI was significantly lower in the upright 5 -g viscous $(P<0.01)$ and in upright 10 -g super-viscous swallows $(P<0.01)$ than in the supine saline. No significant differences were noted in DCI when testing was performed using viscous and super-viscous substrates in the supine position, when compared to the traditional method (Table 2).

As shown in Table 3, DL was significantly higher in supine 5-g and 7 -g viscous swallows than in supine saline swallows $(P<0.01)$. Upright saline and upright 7-g viscous swallows were also associated with higher DL than supine saline $(P<0.05)$.

With the exception of the supine 10-g super viscous substrate, $\mathrm{PB}$ was noted to be significantly higher in all substrates tested in the

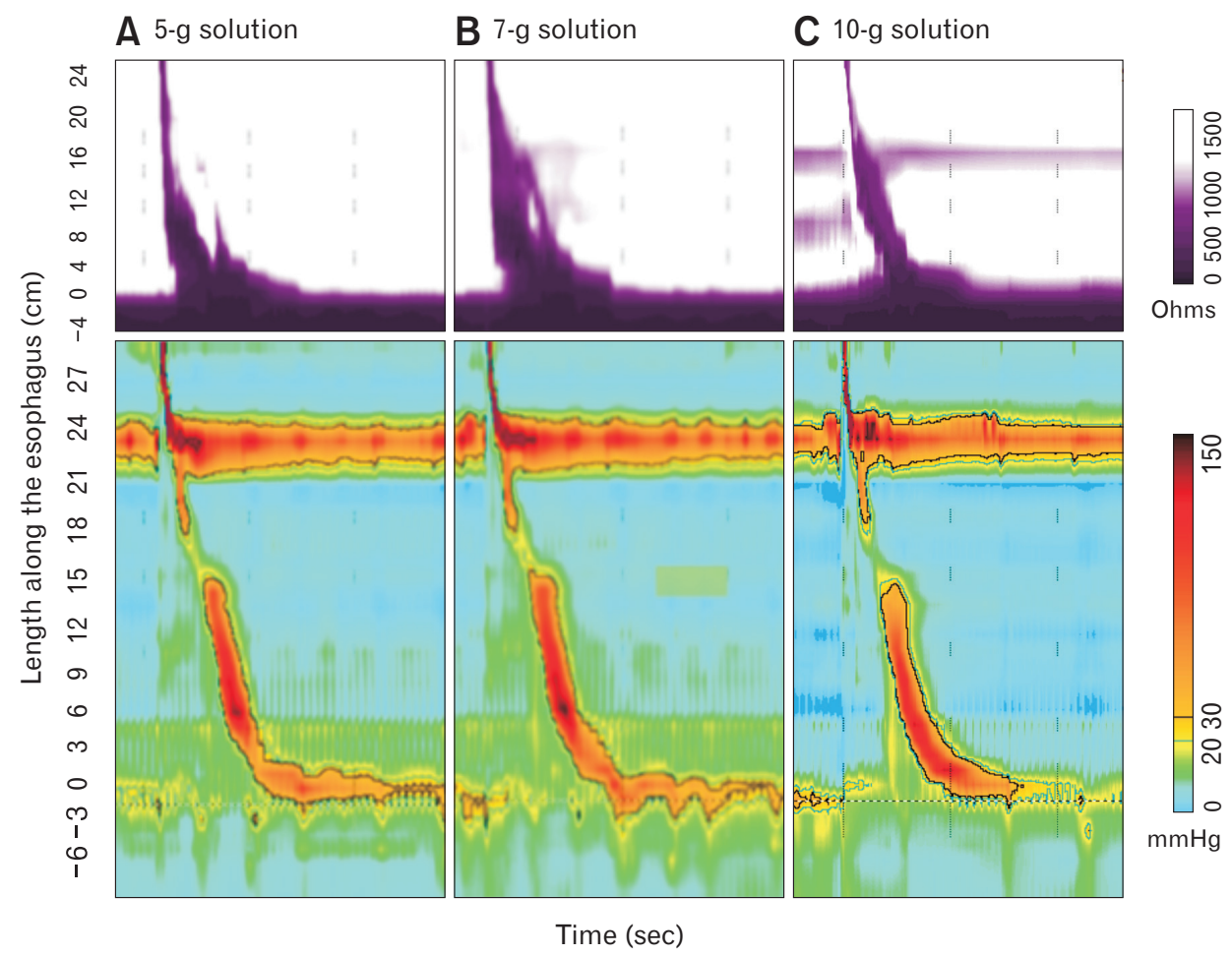

Figure 1. Examples of high-resolution impedance manometry studies using novel viscous and super-viscous solutions. Impedance and high-resolution manometry plots are shown in the top and bottom panels, respectively. (A) 5 -g "Thick-It" solution. (B) 7-g "Thick-It" solution. (C) 10-g "Thick-It" solution. 
Table 1. Integrated Relaxation Pressure Values

\begin{tabular}{|c|c|c|c|c|c|c|c|}
\hline \multirow{2}{*}{ Position/substrate } & \multirow{2}{*}{$\begin{array}{l}\text { Number of } \\
\text { subjects }\end{array}$} & \multirow{2}{*}{$\begin{array}{l}\text { Number of } \\
\text { swallows }\end{array}$} & \multicolumn{5}{|c|}{ IRP } \\
\hline & & & Mean $\pm \mathrm{SD}$ & Median & $5 \%$ & $95 \%$ & $P$-value \\
\hline Supine saline & 18 & 140 & $13.4 \pm 5.0$ & 13.0 & 5.0 & 22.0 & \\
\hline Supine $5 \mathrm{~g}$ & 18 & 90 & $13.2 \pm 4.8$ & 13.5 & 4.5 & 21.0 & 0.581 \\
\hline Supine $7 \mathrm{~g}$ & 8 & 40 & $12.8 \pm 5.7$ & 13.5 & 2.0 & 22.1 & 0.548 \\
\hline Supine $10 \mathrm{~g}$ & 9 & 45 & $13.8 \pm 4.7$ & 14.0 & 7.0 & 21.0 & $<0.01$ \\
\hline Upright saline & 8 & 40 & $7.5 \pm 5.5$ & 7.0 & 0.0 & 18.1 & $<0.01$ \\
\hline Upright $5 \mathrm{~g}$ & 18 & 90 & $9.1 \pm 5.8$ & 8.0 & 2.0 & 20.6 & $<0.01$ \\
\hline Upright $7 \mathrm{~g}$ & 8 & 40 & $9.8 \pm 8.6$ & 9.0 & 1.0 & 29.1 & $<0.01$ \\
\hline Upright $10 \mathrm{~g}$ & 9 & 45 & $9.3 \pm 5.3$ & 9.0 & 3.0 & 15.0 & $<0.01$ \\
\hline
\end{tabular}

IRP, integrated relaxation pressure.

$P$-values represent comparison with supine saline position with paired $t$ tests.

Table 2. Distal Contractile Integral Values

\begin{tabular}{|c|c|c|c|c|c|c|c|}
\hline \multirow{2}{*}{ Position/substrate } & \multirow{2}{*}{$\begin{array}{c}\text { Number of } \\
\text { subjects }\end{array}$} & \multirow{2}{*}{$\begin{array}{c}\text { Number of } \\
\text { swallows }\end{array}$} & \multicolumn{5}{|c|}{ DCI } \\
\hline & & & Mean \pm SD & Median & $5 \%$ & $95 \%$ & $P$-value \\
\hline Supine saline & 18 & 140 & $1891 \pm 1097$ & 1742 & 654.6 & 3907.5 & \\
\hline Supine $5 \mathrm{~g}$ & 18 & 90 & $1815 \pm 1136$ & 1928 & 378.5 & 3774.4 & 0.143 \\
\hline Supine $7 \mathrm{~g}$ & 8 & 40 & $2020 \pm 1717$ & 2207 & 267.7 & 5364.7 & 0.444 \\
\hline Supine $10 \mathrm{~g}$ & 9 & 45 & $1553 \pm 1126$ & 1426 & 68.2 & 3516.4 & 0.834 \\
\hline Upright saline & 8 & 40 & $2519 \pm 2829$ & 2535 & 0.0 & 5311.3 & 0.980 \\
\hline Upright $5 \mathrm{~g}$ & 18 & 90 & $1682 \pm 1663$ & 1401 & 22.8 & 5464.0 & $<0.01$ \\
\hline Upright $7 \mathrm{~g}$ & 8 & 40 & $1721 \pm 1378$ & 1797 & 32.4 & 4697.1 & 0.070 \\
\hline Upright $10 \mathrm{~g}$ & 9 & 45 & $1259 \pm 1295$ & 967 & 56.6 & 3055.4 & $<0.01$ \\
\hline
\end{tabular}

DCI, distal contractile integral.

$P$-values represent comparison with supine saline position with paired $t$ tests.

Table 3. Distal Latency Values

\begin{tabular}{|c|c|c|c|c|c|c|c|}
\hline \multirow{2}{*}{ Position/substrate } & \multirow{2}{*}{$\begin{array}{c}\text { Number of } \\
\text { subjects }\end{array}$} & \multirow{2}{*}{$\begin{array}{l}\text { Number of } \\
\text { swallows }\end{array}$} & \multicolumn{5}{|c|}{$\mathrm{DL}$} \\
\hline & & & Mean $\pm \mathrm{SD}$ & Median & $5 \%$ & $95 \%$ & $P$-value \\
\hline Supine saline & 18 & 140 & $6.8 \pm 1.4$ & 6.7 & 5.0 & 8.8 & \\
\hline Supine $5 \mathrm{~g}$ & 18 & 90 & $7.5 \pm 1.8$ & 7.6 & 5.3 & 10.5 & $<0.01$ \\
\hline Supine $7 \mathrm{~g}$ & 8 & 40 & $7.5 \pm 2.3$ & 7.8 & 5.3 & 10.9 & $<0.01$ \\
\hline Supine $10 \mathrm{~g}$ & 9 & 45 & $7.0 \pm 2.7$ & 6.8 & 1.0 & 10.2 & 0.422 \\
\hline Upright saline & 8 & 40 & $8.6 \pm 4.8$ & 7.1 & 5.2 & 16.4 & $<0.05$ \\
\hline Upright $5 \mathrm{~g}$ & 18 & 90 & $7.8 \pm 1.6$ & 7.6 & 5.5 & 10.0 & 0.086 \\
\hline Upright $7 \mathrm{~g}$ & 8 & 40 & $7.2 \pm 2.8$ & 7.6 & 0 & 10.5 & $<0.05$ \\
\hline Upright $10 \mathrm{~g}$ & 9 & 45 & $8.1 \pm 2.3$ & 7.8 & 5.5 & 12.4 & 0.169 \\
\hline
\end{tabular}

DL, distal latency.

* $P$-values represent comparison with supine saline position with paired $t$ tests.

upright position $(P<0.01)$ (Table 4$)$. $\mathrm{PB}$ in subjects tested with 5 -g and 7-g substrates in the supine position was also higher than that for supine saline testing $(P<0.01)$.
In both the supine and upright positions, impedance was significantly lower with $5-\mathrm{g}, 7-\mathrm{g}$, and 10 -g substrates than with saline in the supine position (Table 5). 
Table 4. Peristaltic Break Values

\begin{tabular}{|c|c|c|c|c|c|c|c|}
\hline \multirow{2}{*}{ Position/substrate } & \multirow{2}{*}{$\begin{array}{l}\text { Number of } \\
\text { subjects }\end{array}$} & \multirow{2}{*}{$\begin{array}{l}\text { Number of } \\
\text { swallows }\end{array}$} & \multicolumn{5}{|c|}{$\mathrm{PB}$} \\
\hline & & & Mean $\pm \mathrm{SD}$ & Median & $5 \%$ & $95 \%$ & $P$-value \\
\hline Supine saline & 18 & 140 & $0.8 \pm 1.8$ & 0.0 & 0 & 3.0 & \\
\hline Supine $5 \mathrm{~g}$ & 18 & 90 & $1.4 \pm 2.2$ & 0.0 & 0 & 4.6 & $<0.01$ \\
\hline Supine $7 \mathrm{~g}$ & 8 & 40 & $2.3 \pm 3.1$ & 0.5 & 0 & 10.1 & $<0.01$ \\
\hline Supine $10 \mathrm{~g}$ & 9 & 45 & $1.6 \pm 2.6$ & 0.0 & 0 & 7.4 & 0.278 \\
\hline Upright saline & 8 & 40 & $2.3 \pm 2.8$ & 0.5 & 0 & 7.5 & $<0.01$ \\
\hline Upright $5 \mathrm{~g}$ & 18 & 90 & $2.8 \pm 3.6$ & 1.0 & 0 & 11.9 & $<0.01$ \\
\hline Upright $7 \mathrm{~g}$ & 8 & 40 & $2.5 \pm 2.7$ & 0.0 & 0 & 7.1 & $<0.01$ \\
\hline Upright $10 \mathrm{~g}$ & 9 & 45 & $4.8 \pm 4.2$ & 3.0 & 2.0 & 9.0 & $<0.01$ \\
\hline
\end{tabular}

$\mathrm{PB}$, peristaltic break.

$P$-values represent comparison with supine saline position with paired $t$ tests.

Table 5. Impedance Values

\begin{tabular}{lcccc}
\hline Position/substrate & $\begin{array}{c}\text { Number } \\
\text { of } \\
\text { subjects }\end{array}$ & $\begin{array}{c}\text { Number } \\
\text { of } \\
\text { swallows }\end{array}$ & $\begin{array}{c}\text { Impedance } \\
(\% \text { complete) }\end{array}$ & $P$-value \\
\hline Supine saline & 18 & 140 & $95 \%$ & \\
Supine 5 g & 18 & 90 & $85 \%$ & $<0.01$ \\
Supine 7 g & 8 & 40 & $88 \%$ & $<0.05$ \\
Supine $10 \mathrm{~g}$ & 9 & 45 & $71 \%$ & $<0.01$ \\
Upright saline & 8 & 40 & $88 \%$ & 0.322 \\
Upright $5 \mathrm{~g}$ & 18 & 90 & $82 \%$ & $<0.05$ \\
Upright 7 g & 8 & 40 & $88 \%$ & $<0.05$ \\
Upright $10 \mathrm{~g}$ & 9 & 45 & $60 \%$ & $<0.01$ \\
\hline
\end{tabular}

$P$-values represent comparison with supine saline position with paired $t$ tests.

\section{Discussion}

In our study, we examined HRiM values using standardized viscous and super-viscous substrates on 18 healthy subjects in supine and upright positions. These parameters were compared to the traditional method of testing with saline in the supine position. We found that position changes (upright vs supine) affected HRiM Chicago metrics. Testing while upright significantly reduced the IRP with saline and with all viscous substrates, including 5-g, 7-g, and $10-\mathrm{g}$. The DCI was also significantly lower when subjects were tested upright using the 5 -g and the $10-\mathrm{g}$ substrates when compared to the traditional method of using saline supine. These results are generally concordant with a study by Zhang et al $^{15}$ wherein 21 healthy volunteers and 25 patients received ten $5-\mathrm{mL}$ liquid swallows and 5 "steamed bread" swallows in both supine and upright positions. Changing to upright position from supine position lowered DCI and IRP. When comparing liquid to solid swallows, solid swallows exhibited more vigorous esophageal contractions (increased DCI). ${ }^{15}$ Also, Xiao et al ${ }^{16}$ performed HRiM on 75 healthy volunteers who received liquid swallows in both supine and upright positions and showed that DCI in upright position was dramatically reduced, when comparing to DCI in supine position.

In our cohort, we found that testing with the $10-\mathrm{g}$ substrate in the supine position significantly increased the IRP when compared to saline in the supine position. Xiao et al ${ }^{16}$ studied 148 patients with a range of complaints. All patients underwent 5 supine and 5 upright liquid swallows. Seventy-five of these 148 patients then underwent an upright "provocative swallow protocol" consisting of five $5-\mathrm{mL}$ liquid swallows, two $10-\mathrm{mL}$ liquid swallows, two 5-mL apple compote swallows, and 2 small marshmallow swallows. Interestingly, 11 patients demonstrated enhanced evidence of esophagogastric junction outflow obstruction with provocative swallows when conventional liquid swallows demonstrated normal or high normal range IRPs. ${ }^{12}$ Three of these patients were treated with endoscopic measures to the lower esophageal sphincter with relief of dysphagia. ${ }^{12}$ Therefore, in addition to evaluating bolus transit, ${ }^{17}$ testing with different substrate viscosity may improve sensitivity of esophageal manometry. The ability to diagnose esophagogastric junction outflow obstruction is important as this diagnosis may be targeted therapeutically.

Compared to liquid swallows, solid food and viscous swallows are thought to be more sensitive to diagnose motility abnormalities. In 1990, Sears et $\mathrm{al}^{11}$ noted that solid swallows increased the frequency of simultaneous/non-peristaltic swallows while upright positioning appeared to decrease esophageal peristaltic amplitudes. This was followed by an observation by Allen et $\mathrm{al}^{14}$ in 1992 that sold food boluses could elicit simultaneous swallows in patients with non-cardiac chest pain. 
In 2008, Bernhard et $\mathrm{al}^{10}$ showed that high-resolution manometry patterns changed in patients with chest pain when upright bread swallows are administered compared to standard supine saline swallows. They concluded that evaluating bread swallows in the upright position reveals motility abnormalities overlooked by saline swallows alone. This study was performed before institution of the Chicago classification and thus used conventional manometry measurements to define motility changes despite using high-resolution equipment. Changing to upright position reduces DCI likely due to the aid of gravity. Using solid food boluses seems to increase DCI as the esophagus works to meet the demand of propagating a thicker bolus. Conflicting findings exist for IRP and DL, but most studies show a trend towards increased DL with solid or viscous swallows. In our study, compared with supine saline swallows, we observed significantly higher DL values in supine 5 -g and 7 -g viscous swallows $(P<$ $0.01)$, as well as higher PB values in subjects tested with 5 -g and 7 -g substrates in the supine position $(P<0.01)$. We did not see differences in IRP and DCI between supine viscous swallows and supine saline swallows.

There are several limitations to using solid boluses in HRiM studies. In an editorial titled "Use of solid boluses in high-resolution manometry," Wang and Sifrim ${ }^{18}$ commented on inter-study discrepancies between the high-resolution metrics. These authors noted that during solid boluses the extent to which the patient chews the food and the proportion of the prescribed food swallowed may vary. Moreover, because these studies were conducted in China, the United States, and Europe, variations in patient demographics may have contributed to different results. The editorial concluded that the differences in previous studies "highlights the need for a standardized protocol and the potential need for localized normative value, as is the case in other gastrointestinal motility investigations. ${ }^{18}$ No studies used the Diversateck's proprietary viscous substrate that is manufactured and standardized for their solid food swallow protocols. In our study, we developed novel standardized viscous and super-viscous substrates that are easy to make and are economical.

In addition to standardization difficulties between previous studies, impedance changes have been largely ignored. As impedance changes are the measure of true bolus transit through the esophagus (as opposed to manometric pressures generated during esophageal contractions), this parameter should be focused on as much as manometry. ${ }^{19-22}$ Few studies incorporated impedance into their upright position analyses. A recent study by Park et al $\mathrm{al}^{23}$ evaluated the effect of upright saline swallows on impedance changes as well as their correlation to peristaltic breaks. They used patients who swallowed saline in an upright position and concluded that peristaltic breaks and incomplete bolus transit do correlate. However, the authors did not establish normal values and did not use viscous swallows. In our study, we showed that, in both supine and upright positions, impedance with our novel viscous and super-viscous solutions was significantly lower than with saline in the supine position.

Limitations of our study include the following: (1) small sample size. To establish normative values of these novel viscous and super-viscous solutions, larger healthy populations based on power calculations will be required; (2) 3 subjects experienced oropharyngeal transfer difficulties on the 10-g super-viscous substrate, therefore, for studies using $10-\mathrm{g}$ substrates, patients will need to be taught proper swallowing techniques to ensure appropriate oropharyngeal transfer prior to initiation of swallows.

In conclusion, we developed novel standardized viscous and super-viscous substrates in a pilot study that are both safe and widely available, and demonstrated the feasibility of using these substrates in HRiM studies. In future studies, we plan to establish normative HRiM values using a larger number of healthy subjects and also evaluate patients with motility disorders and determine whether these viscous and super-viscous substrates improve either diagnostic sensitivity and/or treatment outcomes.

Financial support: This work was supported by a grant to Erik B Person and Guofeng Xie from the Castell Foundation (Grant No. 10015806).

Conflicts of interest: None.

Author contributions: Erik B Person, Uni Wong, and Guofeng Xie performed the research; Guofeng Xie and Erik B Person designed the study; Donald O Castell, Erik von Rosenvinge, and Jean-Pierre Raufman contributed essential tools and to regulatory approvals; Uni Wong analyzed the data; and Guofeng Xie wrote the initial draft and all authors contributed to and approved the final paper.

\section{References}

1. Pandolfino JE, Kahrilas PJ; American Gastroenterological Association. AGA technical review on the clinical use of esophageal manometry. Gastroenterology 2005;128:209-224.

2. van Hoeij FB, Bredenoord AJ. Clinical application of esophageal highresolution manometry in the diagnosis of esophageal motility disorders. J Neurogastroenterol Motil 2016;22:6-13. 
3. Herregods TV, Roman S, Kahrilas PJ, Smout AJ, Bredenoord AJ. Normative values in esophageal high-resolution manometry. Neurogastroenterol Motil 2015;27:175-187.

4. Bredenoord AJ, Fox M, Kahrilas PJ, et al. Chicago classification criteria of esophageal motility disorders defined in high resolution esophageal pressure topography. Neurogastroenterol Motil 2012;24(suppl 1):57-65.

5. Kahrilas PJ, Bredenoord AJ, Fox M, et al. The Chicago classification of esophageal motility disorders, v3.0. Neurogastroenterol Motil 2015;27:160-174.

6. Pandolfino JE, Fox MR, Bredenoord AJ, Kahrilas PJ. High-resolution manometry in clinical practice: utilizing pressure topography to classify oesophageal motility abnormalities. Neurogastroenterol Motil 2009;21:796-806.

7. Schlottmann F, Herbella FA, Patti MG. Understanding the Chicago classification: from tracings to patients. J Neurogastroenterol Motil 2017;23:487-494.

8. Tutuian R, Vela MF, Balaji NS, et al. Esophageal function testing with combined multichannel intraluminal impedance and manometry: multicenter study in healthy volunteers. Clin Gastroenterol Hepatol 2003;1:174-182.

9. Clayton SB, Rife C, Kalbfleisch JH, Castell DO. Viscous impedance is an important indicator of abnormal esophageal motility. Neurogastroenterol Motil 2013;25:563-e455.

10. Bernhard A, Pohl D, Fried M, Castell DO, Tutuian R. Influence of bolus consistency and position on esophageal high-resolution manometry findings. Dig Dis Sci 2008;53:1198-1205.

11. Sears VW Jr, Castell JA, Castell DO. Comparison of effects of upright versus supine body position and liquid versus solid bolus on esophageal pressures in normal humans. Dig Dis Sci 1990;35:857-864.

12. Xiao Y, Nicodème F, Kahrilas PJ, Roman S, Lin Z, Pandolfino JE. Optimizing the swallow protocol of clinical high-resolution esophageal manometry studies. Neurogastroenterol Motil 2012;24:e489-e496.

13. Ang D, Misselwitz B, Hollenstein M, et al. Diagnostic yield of highresolution manometry with a solid test meal for clinically relevant, symp- tomatic oesophageal motility disorders: serial diagnostic study. Lancet Gastroenterol Hepatol 2017;2:654-661.

14. Allen ML, Mellow MH, Robinson M. Manometry during food ingestion aids in the diagnosis of diffuse esophageal spasm. Am J Gastroenterol 1992;87:568-571.

15. Zhang X, Xiang X, Tu L, Xie X, Hou X. Esophageal motility in the supine and upright positions for liquid and solid swallows through highresolution manometry. J Neurogastroenterol Motil 2013;19:467-472.

16. Xiao Y, Read A, Nicodème F, Roman S, Kahrilas PJ, Pandolfino JE. The effect of a sitting vs supine posture on normative esophageal pressure topography metrics and Chicago classification diagnosis of esophageal motility disorders. Neurogastroenterol Motil 2012;24:e509-e516.

17. Zheng E, Gideon RM, Sloan J, Katz PO. Esophagogastric junction outflow obstruction is often associated with coexistent abnormal esophageal body motility and abnormal bolus transit. Dis Esophagus 2017;30:1-4.

18. Wang YT, Sifrim D. Use of solid boluses in high-resolution manometry. J Neurogastroenterol Motil 2013;19:422-423.

19. Nguyen HN, Domingues GR, Winograd R, et al. Impedance characteristics of normal oesophageal motor function. Eur J Gastroenterol Hepatol 2003;15:773-780.

20. Tutuian R, Castell DO. Clinical applications of esophageal multichannel intraluminal impedance testing. Gastroenterol Hepatol (NY) 2006;2:250-258.

21. Kessing BF, Smout AJ, Bredenoord AJ. Clinical applications of esophageal impedance monitoring and high-resolution manometry. Curr Gastroenterol Rep 2012;14:197-205.

22. Bulsiewicz WJ, Kahrilas PJ, Kwiatek MA, Ghosh SK, Meek A, Pandolfino JE. Esophageal pressure topography criteria indicative of incomplete bolus clearance: a study using high-resolution impedance manometry. Am J Gastroenterol 2009;104:2721-2728.

23. Park EJ, Lee JS, Lee TH, et al. High-resolution impedance manometry criteria in the sitting position indicative of incomplete bolus clearance. $\mathrm{J}$ Neurogastroenterol Motil 2014;20:491-496. 